\title{
Rat model of nonalcoholic steatohepatitis
} created by methionine and choline deficiency: biochemical and histological analyses

This article was published in the following Dove Press journal:

Veterinary Medicine: Research and Reports

I 5 July 201 I

Number of times this article has been viewed

\section{Shinichi Nagai' \\ Jun Iwamoto 2 \\ Masakazu Suzuki' \\ Azusa Seki'}

'Hamri Co Ltd, Koga, Ibaraki, Japan; ${ }^{2}$ Institute for Integrated Sports Medicine, Keio University School of Medicine, Shinjuku-ku, Tokyo, Japan
Correspondence: Jun Iwamoto Institute for Integrated Sports Medicine, Keio University School of Medicine, 35 Shinanomachi, Shinjuku-ku,

Tokyo 160-8582, Japan

$\mathrm{Tel}+8 \mid 33353$ | 2 | |

Fax +81333529467

Email jiwamoto@a8.keio.jp
Background: The purpose of this study was to establish a Sprague-Dawley rat model of nonalcoholic steatohepatitis (NASH) due to combined methionine and choline deficiency (MCD).

Methods: Eighty nine-week-old male Sprague-Dawley rats were randomized into two groups $(\mathrm{n}=40)$, comprising an MCD diet group and a standard diet (control) group. After fasting blood was collected, 10 rats from each group were scheduled to be sacrificed at weeks 4, 8, 12, and 16 from the start of the experiment. Body weight and liver wet weight were measured, and histological examination of the liver was performed after hematoxylin and eosin and Oil Red O staining. Results: In the MCD group, body weight and liver wet weight were decreased compared with the control group, while serum levels of albumin, $\gamma$-glutamyltranspeptidase, alkaline phosphatase, and total bilirubin were increased, but serum levels of total cholesterol and triglycerides were decreased. Histological examination of the liver revealed centrilobular hepatocellular fatty change from as early as four weeks, with mild fibrosis after 12 weeks.

Conclusion: These findings suggested the onset of NASH with liver dysfunction and bile duct damage in rats fed with the MCD diet. Increased fatty acid uptake and decreased cholesterol secretion were considered to be important mechanisms by which the MCD diet promoted intrahepatic lipid accumulation in this model.

Keywords: nonalcoholic steatohepatitis, rat, methionine, choline, fatty liver

\section{Introduction}

Nonalcoholic fatty liver disease is one of the most common chronic liver disorders, and covers a broad spectrum, ranging from simple steatosis to cirrhosis. ${ }^{1-3}$ Nonalcoholic steatohepatitis (NASH) is the part of this spectrum that forms the borderline between the benign condition of steatosis and the serious condition of cirrhosis. NASH occurs in patients without significant alcohol consumption, although it resembles alcoholic liver disease histologically. ${ }^{2,4}$ Recent reports have indicated that patients with fatty liver are more susceptible to developing cirrhosis and hepatocellular carcinoma, suggesting that fatty liver is not actually benign. ${ }^{5}$

The mechanisms involved in progression from a normal liver to NASH have been clarified in various experimental models, such as animals with genetic hyperphagia, animals on methionine and choline deficiency (MCD) diets, animals treated with pharmacological agents that inhibit lipoprotein synthesis and secretion, and animals fed a high-fat diet. ${ }^{6-11}$ Histologically, NASH is defined by the presence of steatosis, lobular inflammation, hepatocellular injury (ballooning degeneration), and progression to hepatic fibrosis. ${ }^{2}$ submit your manuscript | www.dovepress.com

Dovepress

http://dx.doi.org// 0.2147/VMRR.S22205
Veterinary Medicine: Research and Reports 20I I:2 I-8

(C) 20I I Nagai et al, publisher and licensee Dove Medical Press Ltd. This is an Open Access article which permits unrestricted noncommercial use, provided the original work is properly cited. 
Various rodent models have contributed to our understanding of the histopathology of NASH. Mice are suitable for molecular biology analysis, whereas rats are commonly used for biochemical analysis. The Wistar strain may be associated with the greatest degree of NASH,${ }^{11-13}$ but biochemical and histological changes have not been fully examined in the Sprague-Dawley strain. MCD deficiency is known to induce NASH in mice. ${ }^{14}$ Accordingly, the present study was performed to establish a Sprague-Dawley rat model of NASH due to MCD, followed by both biochemical and histological characterization.

\section{Materials and methods}

\section{Animal model}

Eighty eight-week-old male Sprague-Dawley rats were purchased from Charles River Laboratories Japan (Kanagawa, Japan). The animals were housed (two rats per cage) in an animal room (temperature $24^{\circ} \mathrm{C}$, humidity $50 \%$, and a 12-hour on/off light cycle) with free access to water and a chow diet. After allowing one week for adaptation to the new environment, the nine-week-old rats were randomized by the stratified weight method into two groups $(n=40)$ that received either an MCD diet (Research Diets Inc, New Brunswick, NJ) or a standard diet (Research Diets Inc). The MCD diet contained neither methionine nor choline, while the standard diet contained $5.1 \mathrm{~g}$ methionine per $\mathrm{kg}$ and $2.5 \mathrm{~g}$ choline per kg. These groups were designated the MCD group and the control group, respectively. Body weight was monitored weekly. Food intake was assessed twice a week using the following formula: (the amount of chow diet supplied during a 24-hour period - the amount of chow diet remaining during the same period in a cage)/2, because two rats were housed in a cage. The total duration of the experiment was 16 weeks. This experiment was carried out at the laboratory of Hamri Co Ltd (Ibaraki, Japan), which is approved by the Association for the Assessment and Accreditation of Laboratory Animal Care. The experimental protocol was approved by the Institutional Animal Care and Use Committee of Hamri Co Ltd (Ibaraki, Japan).

\section{Preparation of specimens}

The animals to be sampled at weeks $4,8,12$, and 16 after the start of the experiment (10 animals per group) were randomly grouped at baseline. After fasting for 12 hours, blood was scheduled to be collected and the animals were planned to be sacrificed by exsanguination after being anesthetized via inhalation of 2\%-3\% isoflurane (Mylan Inc, Tokyo, Japan) using a Table Top Laboratory Animal Anesthesia System (V1 Type
VetEquip Inc, CA) at weeks 4, 8, 12, and 16 after the start of the experiment. However, two rats from the control group died before 12 weeks and one rat each from the control and MCD groups died before 16 weeks. Necropsies by veterinarians did not show any clear etiology of death in these four rats. After sacrifice, the liver was isolated from each animal. Blood samples were processed for biochemical analysis, while the livers were kept in $10 \%$ neutral buffered formalin and processed for histological analysis after the wet weight was measured with an electronic balance (A and D Company, Tokyo, Japan).

\section{Analysis of biochemical parameters}

Serum levels of total protein, albumin, albumin/globulin ratio, aspartate aminotransferase, alanine aminotransferase, gamma-glutamyltranspeptidase, alkaline phosphatase, total bilirubin, direct bilirubin, total cholesterol, and triglycerides were measured by standard techniques.

\section{Histological examination of liver}

Formalin-fixed and paraffin-embedded liver tissue was cut into $4 \mu \mathrm{m}$ sections and stained with hematoxylin and eosin and Oil Red O stains. Histological examination was performed under an Olympus light microscope (Olympus, Tokyo, Japan). The hematoxylin and eosin-stained sections were used to assess hepatocellular fatty change, fibrosis, inflammatory cells, microgranulomas, congestion, and hepatocyte necrosis, while Oil red O staining was employed to detect fat. ${ }^{15-17}$

\section{Statistical analysis}

Results are expressed as means and standard deviation. Before comparison of biochemical data between the two groups, the F-test was used to assess the homogeneity of variance. If the variance was homogeneous, the Student's $t$-test was applied. If the variance was not homogeneous, Welch's test was applied. Comparison of liver histological scores was performed using Fisher's Exact test. All statistical analyses were done with Excel Analysis 2008 Software (Social Survey Research Information Co Ltd, Tokyo, Japan) on a Windows computer. Significance was accepted at $P<0.05$ for all analyses.

\section{Results}

\section{Body weight and food intake}

Figure 1 shows that body weight continued to increase in the control group, but gradually decreased in the MCD group. After seven days, body weight was significantly lower in the MCD group than in the control group. 


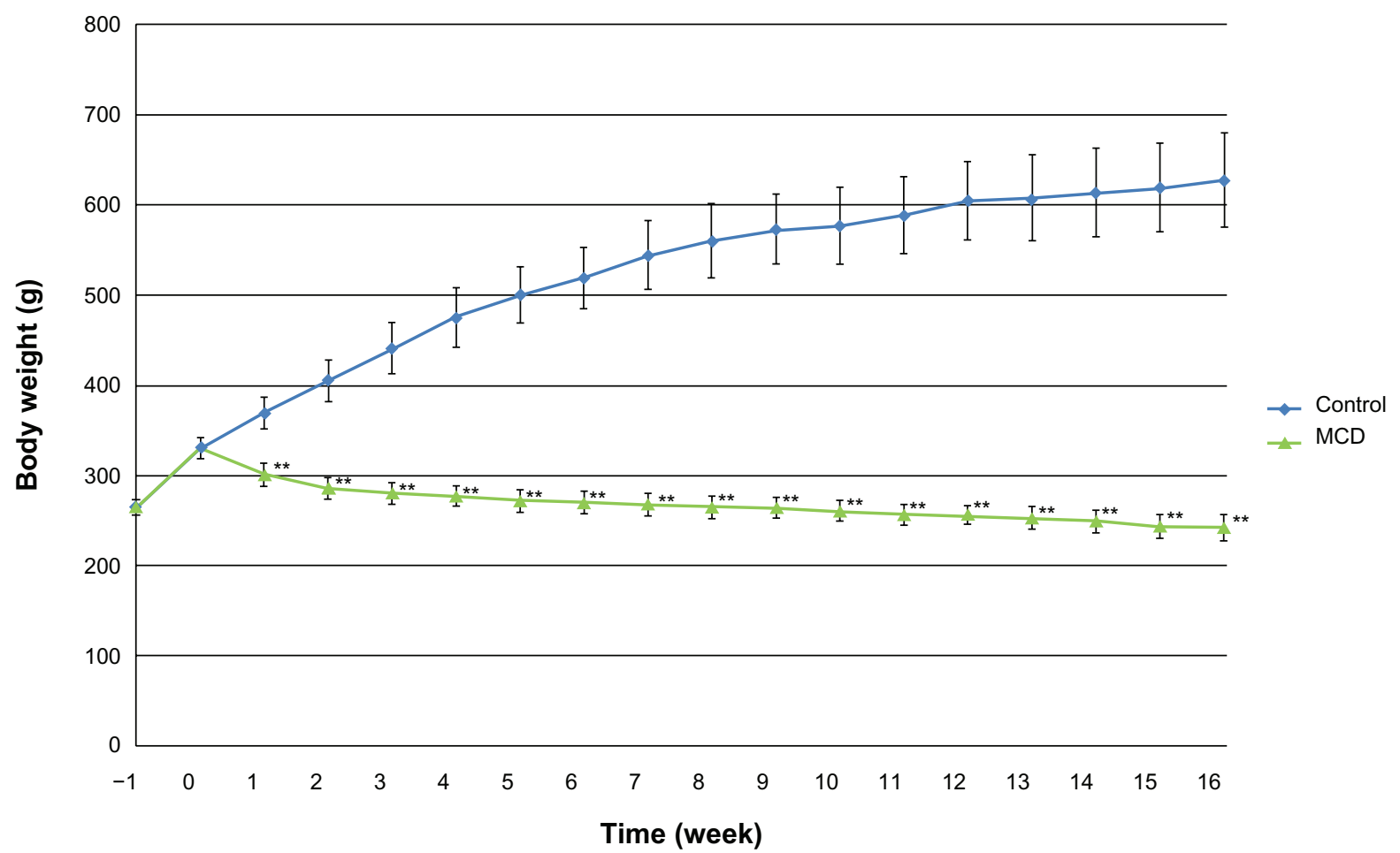

Figure I Body weight of the MCD and control groups during the I6-week study period.

Notes: Data are expressed as means \pm standard deviation. Body weight of the rats was measured weekly. Weight significantly decreased in the MCD group compared with the control group. $* * P<0.001$ vs control.

Abbreviation: MCD, methionine and choline deficiency.

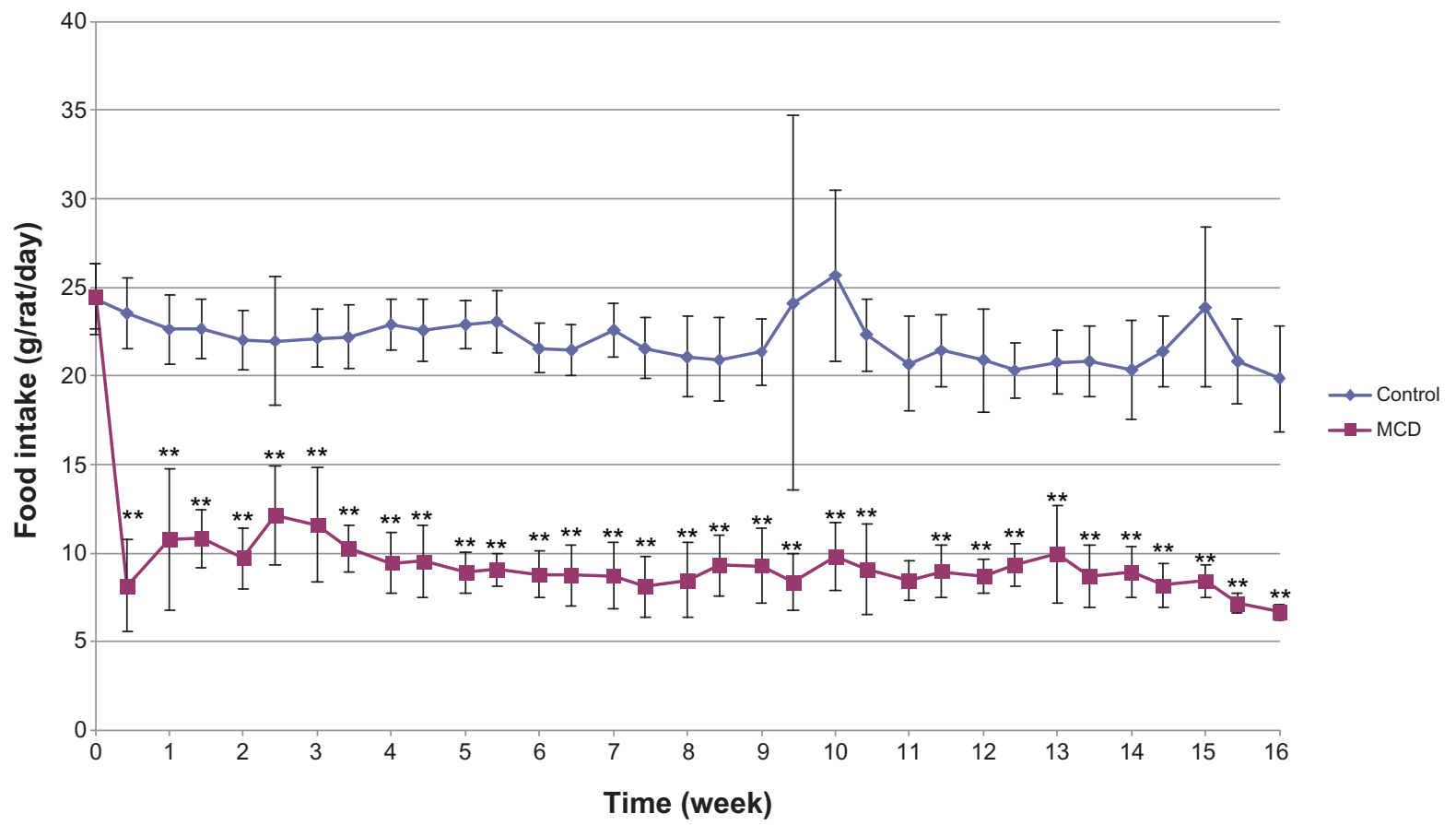

Figure 2 Food intake of the MCD and control groups during the 16-week study period.

Notes: Data are expressed as means \pm standard deviation. Food intake was assessed twice a week using the following formula: (amount of chew diet supplied during 24-hour period - amount of chow diet that remained during the same period in a cage)/2, because two rats were housed in a cage. Food intake was significantly decreased in the MCD group compared with the control group. $* * P<0.00$ I vs control.

Abbreviation: MCD, methionine and choline deficiency. 
Figure 2 shows that daily food intake was sustained during the 16-week period in the control group, but rapidly decreased in several days and was persistant thereafter in the MCD group. After several days, daily food intake was significantly lower in the MCD group than in the control group.

\section{Serum biochemical parameters}

Figure 3 shows that the serum total cholesterol and triglyceride levels were significantly lower and the albu$\mathrm{min} / \mathrm{globulin}$ ratio was significantly higher after four weeks in the MCD group than in the control group. Serum $\gamma$-glutamyltranspeptidase and total bilirubin levels were significantly higher after eight weeks in the MCD group than in the control group. Serum albumin levels were significantly higher at weeks 4, 8, and 16 in the MCD group than in the control group. Serum alanine aminotransferase levels were significantly lower at weeks 4,8 , and 12 in the MCD group than in the control group, but did not differ significantly at 16 weeks between the two groups. Serum total protein, aspartate aminotransferase, and direct bilirubin levels did not differ significantly between the two groups during the 16-week study period.

\section{Liver histology}

Table 1 shows the results obtained by histological examination of the liver. Centrilobular hepatocellular fatty change was not observed during the 16 weeks in the control group, but was found from as early as four weeks and persisted until 16 weeks in the MCD group. Mild fibrosis was not observed during the 16 weeks in the control group, but was found after 12 weeks in the MCD group. Diffuse hepatocellular fatty change was not observed during the 16-week period in the MCD group, but was found at four and eight weeks in the control group. Periportal hepatocellular fatty change was not observed after 12 weeks in the MCD group, but was found at 12 weeks in the control group.
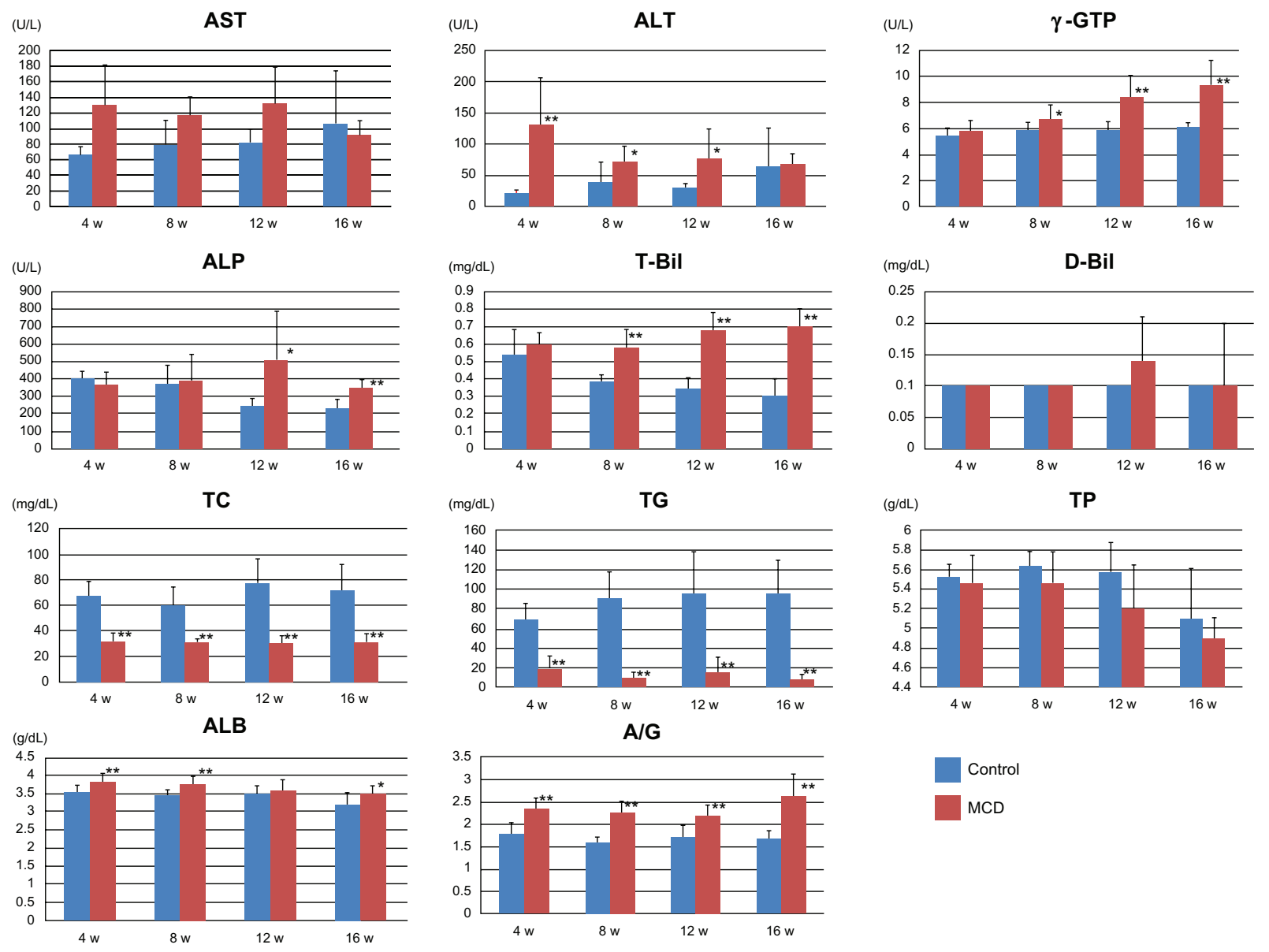

Figure 3 Biochemical markers of the MCD and control groups at weeks 4, 8, 12, and 16 after the start of the experiment.

Notes: Data are expressed as means \pm standard deviation. In the MCD group, serum levels of albumin, $\gamma$-glutamyltranspeptidase, alkaline phosphatase, and total bilirubin were increased compared with those in the control group. Conversely, serum levels of total cholesterol and triglycerides were decreased. $* P<0.05$ vs control; $* * P<0.00 \mathrm{I}$ vs control. Abbreviations: AST, aspartate aminotransferase; ALT, alanine aminotransferase; $\gamma$-GTP, $\gamma$-glutamyltranspeptidase; ALP, alkaline phosphatase; T-Bil, total bilirubin; D-Bil, direct bilirubin; TC, total cholesterol; TG, triglyceride; TP, total protein; ALB, albumin; A/G, albumin/globulin; MCD, methionine and choline deficiency. 
Table I Histopathology: incidence of microscopic lesions

\begin{tabular}{|c|c|c|c|c|c|c|c|c|}
\hline \multirow[b]{2}{*}{ After 4 weeks } & \multicolumn{4}{|c|}{ Control $(n=10)$} & \multicolumn{4}{|c|}{$\operatorname{MCD}(n=10)$} \\
\hline & & & & & & & & \\
\hline Grade & - & + & ++ & +++ & - & + & ++ & +++ \\
\hline \multicolumn{9}{|l|}{ Hematoxylin-Eosin } \\
\hline Diffuse hepatocellular fatty change & 6 & 4 & 0 & 0 & 10 & 0 & 0 & $0^{*}$ \\
\hline Periportal hepatocellular fatty change & 0 & 0 & 0 & 0 & 0 & 0 & 0 & 0 \\
\hline Centrilobular hepatocellular fatty change & 10 & 0 & 0 & 0 & 0 & 0 & 0 & $10 * *$ \\
\hline Fibrosis & 0 & 0 & 0 & 0 & 0 & 0 & 0 & 0 \\
\hline Inflammatory cell infiltration & 9 & I & 0 & 0 & 10 & 0 & 0 & 0 \\
\hline Centrilobular hepatocyte necrosis & 0 & 0 & 0 & 0 & 0 & 0 & 0 & 0 \\
\hline Microgranulomas & 8 & 2 & 0 & 0 & 10 & 0 & 0 & 0 \\
\hline \multirow[t]{2}{*}{ Oil red $\bigcirc$ positive } & 8 & 2 & 0 & 0 & 0 & 0 & 0 & 10 \\
\hline & \multicolumn{4}{|c|}{ Control $(n=10)$} & \multicolumn{4}{|c|}{$\operatorname{MCD}(n=10)$} \\
\hline \multicolumn{9}{|l|}{ After 8 weeks } \\
\hline Grade & - & + & ++ & +++ & - & + & ++ & +++ \\
\hline \multicolumn{9}{|l|}{ Hematoxylin-Eosin } \\
\hline Diffuse hepatocellular fatty change & 5 & 4 & $\mathrm{I}$ & 0 & 10 & 0 & 0 & $0^{*}$ \\
\hline Periportal hepatocellular fatty change & 0 & 0 & 0 & 0 & 0 & 0 & 0 & 0 \\
\hline Centrilobular hepatocellular fatty change & 10 & 0 & 0 & 0 & 0 & 0 & 0 & $10 * *$ \\
\hline Fibrosis & 10 & 0 & 0 & 0 & 8 & 2 & 0 & 0 \\
\hline Inflammatory cell infiltration & 10 & 0 & 0 & 0 & 8 & 2 & 0 & 0 \\
\hline Centrilobular hepatocyte necrosis & 9 & 0 & 0 & 1 & 10 & 0 & 0 & 0 \\
\hline Microgranulomas & 9 & I & 0 & 0 & 10 & 0 & 0 & 0 \\
\hline \multirow[t]{2}{*}{ Oil red $O$ positive } & 4 & 6 & 0 & 0 & 0 & 0 & 0 & 10 \\
\hline & \multicolumn{4}{|c|}{ Control $(n=8)$} & \multicolumn{4}{|c|}{$\operatorname{MCD}(n=10)$} \\
\hline \multicolumn{9}{|l|}{ After 12 weeks } \\
\hline Grade & - & + & ++ & +++ & - & + & ++ & +r+ \\
\hline \multicolumn{9}{|l|}{ Hematoxylin-Eosin } \\
\hline Diffuse hepatocellular fatty change & 7 & I & 0 & 0 & 10 & 0 & 0 & 0 \\
\hline Periportal hepatocellular fatty change & 4 & 4 & 0 & 0 & 10 & 0 & 0 & $0 *$ \\
\hline Centrilobular hepatocellular fatty change & 8 & 0 & 0 & 0 & 0 & 0 & 0 & $10 * *$ \\
\hline Fibrosis & 8 & 0 & 0 & 0 & 5 & 5 & 0 & $0^{*}$ \\
\hline Inflammatory cell infiltration & 8 & 0 & 0 & 0 & 7 & 3 & 0 & 0 \\
\hline Centrilobular hepatocyte necrosis & 0 & 0 & 0 & 0 & 0 & 0 & 0 & 0 \\
\hline Microgranulomas & 5 & 3 & 0 & 0 & 10 & 0 & 0 & 0 \\
\hline \multirow[t]{2}{*}{ Oil red $O$ positive } & 5 & 3 & 0 & 0 & 0 & 0 & 0 & 10 \\
\hline & \multicolumn{4}{|c|}{ Control $(n=9)$} & \multicolumn{4}{|c|}{$\operatorname{MCD}(n=9)$} \\
\hline \multicolumn{9}{|l|}{ After 16 weeks } \\
\hline Grade & - & + & ++ & +++ & - & + & ++ & +++ \\
\hline \multicolumn{9}{|l|}{ Hematoxylin-Eosin } \\
\hline Diffuse hepatocellular fatty change & 7 & 2 & 0 & 0 & 9 & 0 & 0 & 0 \\
\hline Periportal hepatocellular fatty change & 5 & 4 & 0 & 0 & 9 & 0 & 0 & 0 \\
\hline Centrilobular hepatocellular fatty change & 9 & 0 & 0 & 0 & 0 & 0 & 0 & $9 * *$ \\
\hline Fibrosis & 9 & 0 & 0 & 0 & 1 & 7 & 1 & $0 * *$ \\
\hline Inflammatory cell infiltration & 9 & 0 & 0 & 0 & 9 & 0 & 0 & 0 \\
\hline Centrilobular hepatocyte necrosis & 6 & 2 & 1 & 0 & 9 & 0 & 0 & 0 \\
\hline Microgranulomas & 6 & 3 & 0 & 0 & 9 & 0 & 0 & 0 \\
\hline Oil red $\mathrm{O}$ positive & 5 & 4 & 0 & 0 & 0 & 0 & 0 & 9 \\
\hline
\end{tabular}

Notes: - , no change; + , slight; ++ , moderate; +++ , severe. The incidence was analyzed by Fisher's exact probability test. $* P<0.05, * * P<0.01$ vs control. Abbreviation: MCD, methionine and choline deficiency.

\section{Representative liver findings}

Figure 4 shows representative histological images of the liver, including normal liver stained with hematoxylin and eosin, hepatocellular fatty change stained with hematoxylin and eosin, and Oil Red O, and hepatocellular fatty change plus moderate fibrosis stained with hematoxylin and eosin, and Oil Red O.

\section{Discussion}

The purpose of the present study was to establish a Sprague-Dawley rat model of NASH created by MCD, with the animals being subjected to both biochemical and histological analyses. Histological examination of rats fed a MCD diet confirmed centrilobular fatty change in the 

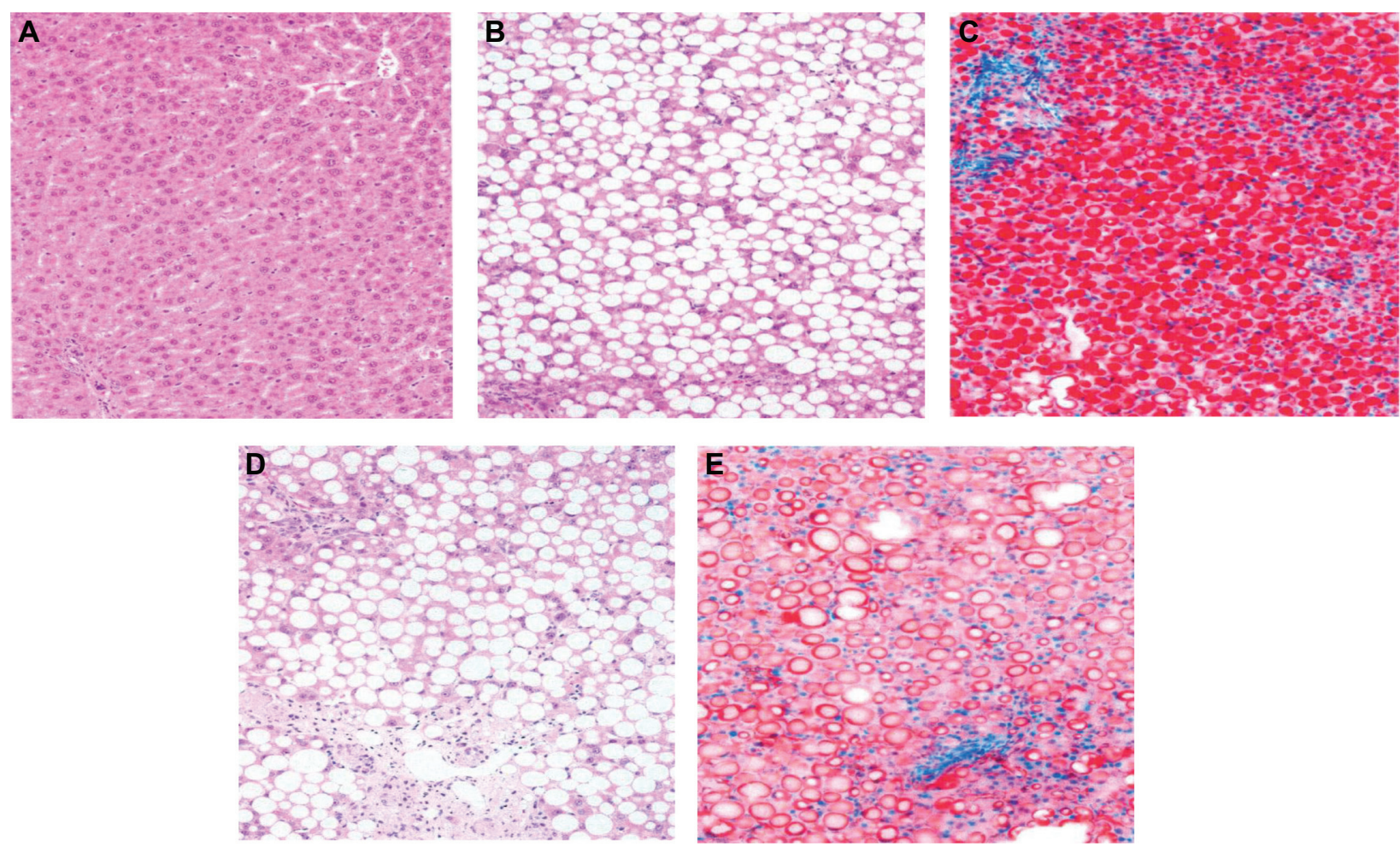

Figure 4 Representative histological images of the liver. (A) No abnormal findings after four weeks in rats fed a standard diet. The liver was stained with hematoxylin and eosin (magnification $\times 160$ ). (B) Centrilobular hepatocellular fatty change after four weeks in rats fed an MCD diet. The liver was stained with hematoxylin and eosin (magnification $\times 160$ ). (C) Centrilobular hepatocellular fatty change after four weeks in rats fed an MCD diet. Staining with Oil Red $O$ (magnification $\times 160$ ). (D) Centrilobular hepatocellular fatty change and moderate fibrosis after 16 weeks in rats fed an MCD diet. The liver was stained with hematoxylin and eosin (magnification $\times 160$ ). (E) Centrilobular hepatocellular fatty change after 16 weeks in rats fed an MCD diet. Staining with Oil Red O (magnification $\times 160$ ).

Abbreviation: MCD, methionine and choline deficiency.

liver from as early as four weeks, with mild fibrosis after 12 weeks. Measurement of serum biochemical parameters showed evidence of liver dysfunction and bile duct damage characterized by increased levels of albumin, $\gamma$-glutamyltranspeptidase, alanine aminotransferase, and total bilirubin in rats fed the MCD diet.

NASH is defined by the presence of steatosis, lobular inflammation, hepatocellular injury (ballooning degeneration), and progression to hepatic fibrosis. ${ }^{2}$ The "two-hit" theory is currently the most widely accepted explanation for progression of NASH. ${ }^{18}$ The first hit is accumulation of fatty acids in the liver caused by several mechanisms (steatosis). ${ }^{18}$ Steatosis involves relatively minor pathological changes and is easily reversible. ${ }^{18}$ However, secondary cellular stress can cause steatosis to progress to steatohepatitis, which is characterized by inflammation and fibrosis. ${ }^{19,20}$ Histological examination of the liver in our rat model of NASH confirmed the presence of both steatosis and moderate fibrosis.

The pathophysiology of NASH caused by MCD has been elucidated using mice fed an MCD diet. Nuclear factor- $\mathrm{\kappa B}$, tumor necrosis factor- $\alpha$, peroxisomal proliferator active receptor- $\gamma, \alpha$-smooth muscle actin, transforming growth factor- $\beta 1$, and connective tissue growth factor have been reported to play a role in the occurrence of NASH. ${ }^{21-23}$ Insulin resistance and oxidative stress have also been suggested to have an association with NASH in rodents fed an MCD diet. ${ }^{13,22-25}$

The MCD diet has been reported to cause accumulation of lipids in the liver and an increase of serum alkaline phosphatase levels in mice, ${ }^{14}$ and the increase of alkaline phosphatase suggests bile duct damage and cholestasis. However, biochemical changes have not been fully examined in previous rodent models of NASH. In our rat model of NASH, both body weight and liver wet weight were decreased. Serum levels of albumin, $\gamma$-glutamyltranspeptidase, alkaline phosphatase, and total bilirubin were increased, suggesting liver dysfunction and bile duct injury, while serum levels of total cholesterol and triglycerides were decreased. Serum alanine aminotransferase levels did not differ significantly at 16 weeks between the MCD and control groups. Alanine aminotransferase may be less responsive to MCD than alkaline phosphatase in rodents, as reported in a previous study. ${ }^{14}$ Increased fatty acid uptake and decreased very low-density lipoprotein secretion were considered to be important mechanisms by which the MCD diet promoted intrahepatic lipid 
accumulation in this model. ${ }^{26}$ This may explain low levels of total cholesterol and triglycerides in blood.

Because of the current epidemics of obesity and diabetes mellitus, ${ }^{27}$ nonalcoholic fatty liver disease has become the most common cause of chronic liver disease. ${ }^{28,29}$ Hossain et al ${ }^{30}$ found that patients with NASH were more likely to be male and have higher levels of alanine aminotransferase, aspartate aminotransferase, and triglycerides, but lower levels of high-density lipoprotein cholesterol, and that male gender, Caucasian ethnicity, diabetes mellitus, and increased levels of aspartate aminotransferase and alanine aminotransferase were predictors of moderate to severe fibrosis. ${ }^{30}$ Poynard et $\mathrm{al}^{31}$ also reported that patients with NASH were older and had higher levels of $\alpha 2$ macroglobulin, aspartate aminotransferase and alanine aminotransferase, glucose, and triglycerides, as well as a higher fibrosis stage and steatosis grade. ${ }^{31}$ There appears to be a discrepancy of biochemical changes, including alanine aminotransferase and triglycerides, between rodents and patients with NASH despite the similar liver histology. Patients with NASH may have fatty liver and release triglycerides from the liver into the circulation, whereas rats with NASH may sequester triglycerides from the blood in the liver. Thus, changes in body weight and serum total cholesterol and triglycerides in our rat model of NASH created by combined MCD did not resemble those in patients with NASH.

One possible explanation for weight loss in the MCD group could be MCD-induced suppression of stearoylcoenzyme A desaturase-1 in the liver, which likely contributes to hypermetabolism and weight loss, and induces hepatic steatosis. ${ }^{32}$ Another possible explanation could be low food intake. Either a deficiency or an excess of methionine has been reported to depress both food consumption and body weight gain in chicks, ${ }^{33}$ suggesting that methionine may control appetite in terms of food intake in animals.

In conclusion, a model of NASH was created by feeding Sprague-Dawley rats an MCD diet for 16 weeks. Histological examination of the liver confirmed the occurrence of centrilobular fatty change and moderate fibrosis. Biochemical analysis showed liver dysfunction and bile duct damage characterized by increased serum levels of albumin, $\gamma$-glutamyltranspeptidase, alkaline phosphatase, and total bilirubin, but decreased serum levels of total cholesterol and triglycerides. We have illustrated the characteristics of a Sprague-Dawley rat model of NASH created by MCD from both the histological and biochemical perspectives. Further studies are needed to reveal the mechanism for the disease in a Sprague-Dawley rat model of NASH.

\section{Disclosure}

The authors report no funding sources or conflict of interest in this work.

\section{References}

1. Farrell GC, Larter CZ. Nonalcoholic fatty liver disease: from steatosis to cirrhosis. Hepatology. 2006;43(2 Suppl 1):S99-S112.

2. Ludwig J, Viggiano TR, McGill DB, et al. Nonalcoholic steatohepatitis: Mayo Clinic experiences with a hitherto unnamed disease. Mayo Clin Proc. 1980;55:434-438.

3. Ludwig J, McGill DB, Lindor KD. Review: nonalcoholic steatohepatitis. J Gastroenterol Hepatol. 1997;12:398-403.

4. Matteoni CA, Younossi ZM, Gramlich T, et al. Nonalcoholic fatty liver disease: a spectrum of clinical and pathological severity. Gastroenterology. 1999;116:1413-1419.

5. Diehl AM. Nonalcoholic fatty liver disease: implications for alcoholic liver disease pathogenesis. Alcohol Clin Exp Res. 2001;25 (5 Suppl ISBRA):8S-14S.

6. Baumgardner JN, Shankar K, Hennings L, et al. A new model for nonalcoholic steatohepatitis in the rat utilizing total enteral nutrition to overfeed a high-polyunsaturated fat diet. Am J Physiol Gastrointest Liver Physiol. 2008;294:G27-G38.

7. Carmiel-Haggai M, Cederbaum AI, Nieto N. A high-fat diet leads to the progression of non-alcoholic fatty liver disease in obese rats. FASEB J. 2005;19:136-138.

8. Deng QG, She H, Cheng JH, et al. Steatohepatitis induced by intragastric overfeeding in mice. Hepatology. 2005;42:905-914.

9. Lettéron P, Sutton A, Mansouri A, et al. Inhibition of microsomal triglyceride transfer protein: another mechanism for drug-induced steatosis in mice. Hepatology. 2003;38:133-140.

10. Lieber CS, Leo MA, Mak KM, et al. Model of nonalcoholic steatohepatitis. Am J Clin Nutr. 2004;79:502-509.

11. Weltman MD, Farrell GC, Liddle C. Increased hepatocyte CYP2E1 expression in a rat nutritional model of hepatic steatosis with inflammation. Gastroenterology. 1996;111:1645-1653.

12. Imeryuz N, Tahan V, Sonsuz A, et al. Iron preloading aggravates nutritional steatohepatitis in rats by increasing apoptotic cell death. J Hepatol. 2007;47:85-89.

13. Tahan V, Eren F, Avsar E, et al. Rosiglitazone attenuates liver inflammation in a rat model of nonalcoholic steatohepatitis. Dig Dis Sci. 2007;52:346-372.

14. Gyamfi MA, Damjanov I, French S, et al. The pathogenesis of ethanol versus methionine and choline deficient diet-induced liver injury Biochem Pharmacol. 2008;75:981-995.

15. de Lima VM, Oliveira CP, Alves VA, et al. A rodent model of NASH with cirrhosis, oval cell proliferation and hepatocellular carcinoma. J Hepatol. 2008;249:105-161.

16. Tasci I, Mas MR, Vural SA, et al. Rat liver fibrosis regresses better with pegylated interferon alpha $2 \mathrm{~b}$ and ursodeoxycholic acid treatments than spontaneous recovery. Liver Int. 2006;26:261-268.

17. Veteläinen R, van Vliet AK, van Gulik TM. Severe steatosis increases hepatocellular injury and impairs liver regeneration in a rat model of partial hepatectomy. Ann Surg. 2007;245:44-50.

18. Day CP, James OF. Steatohepatitis: a tale of two "hits"? Gastroenterology. 1998;114:842-845.

19. Burt AD, Mutton A, Day CP. Diagnosis and interpretation of steatosis and steatohepatitis. Semin Diagn Pathol. 1998;15:246-258.

20. Tokushige K, Takakura M, Tsuchiya-Matsushita N, et al. Influence of TNF gene polymorphisms in Japanese patients with NASH and simple steatosis. J Hepatol. 2007;46:1104-1110.

21. Dela Peña A, Leclercq I, Field J, et al. NF-kappaB activation, rather than TNF, mediates hepatic inflammation in a murine dietary model of steatohepatitis. Gastroenterology. 2005;129:1663-1674.

22. Nan YM, Fu N, Wu WJ, et al. Rosiglitazone prevents nutritional fibrosis and steatohepatitis in mice. Scand J Gastroenterol. 2009;44:358-365. 
23. Nan YM, Wu WJ, Fu N, et al. Antioxidants vitamin E and 1-aminobenzotriazole prevent experimental non-alcoholic steatohepatitis in mice. Scand J Gastroenterol. 2009;44:1121-1131.

24. Sanyal AJ, Campbell-Sargent C, Mirshahi F, et al. Nonalcoholic steatohepatitis: association of insulin resistance and mitochondrial abnormalities. Gastroenterology. 2001;120:1183-1192.

25. George J, Pera N, Phung N, et al. Lipid peroxidation, stellate cell activation and hepatic fibrogenesis in a rat model of chronic steatohepatitis. J Hepatol. 2003;39:756-764.

26. Rinella ME, Elias MS, Smolak RR, et al. Mechanisms of hepatic steatosis in mice fed a lipogenic methionine choline-deficient diet. J Lipid Res. 2008;49:106-176.

27. Mokdad AH, Ford ES, Bowman BA, et al. Prevalence of obesity, diabetes, and obesity-related health risk factors, 2001. JAMA. 2003;289: 76-79.

28. Browning JD, Szczepaniak LS, Dobbins R, et al. Prevalence of hepatic steatosis in an urban population in the United States: impact of ethnicity. Hepatology. 2004;40:1387-1395.
29. Jimba S, Nakagami T, Takahashi M, et al. Prevalence of nonalcoholic fatty liver disease and its association with impaired glucose metabolism in Japanese adults. Diabet Med. 2005;22: 1141-1145.

30. Hossain N, Afendy A, Stepanova M, et al. Independent predictors of fibrosis in patients with nonalcoholic fatty liver disease. Clin Gastroenterol Hepatol. 2009;7:1224-1229.

31. Poynard T, Ratziu V, Charlotte F, et al. Diagnostic value of biochemical markers (NashTest) for the prediction of non alcoholo steato hepatitis in patients with non-alcoholic fatty liver disease. BMC Gastroenterol. 2006;6:34.

32. Rizki G, Arnaboldi L, Gabrielli B, et al. Mice fed a lipogenic methionine-choline-deficient diet develop hypermetabolism coincident with hepatic suppression of SCD-1. J Lipid Res. 2006;47: 228-290.

33. Khalil AA, Thomas OP, Combs GF. Influence of body composition, methionine deficiency or toxicity and ambient temperature on feed intake in the chick. $J$ Nutr. 1968;96:337-341.

\section{Publish your work in this journal}

Veterinary Medicine: Research and Reports is an international, peer-reviewed, open access journal publishing original research, case reports, editorials, reviews and commentaries on all areas of veterinary medicine. The manuscript management system is completely online and includes a very quick and fair peer-review system.
Visit http://www.dovepress.com/testimonials.php to read real quotes from published authors. 